\title{
Formation mechanism of peapod-derived double-walled carbon nanotubes
}

\author{
Feng Ding, ${ }^{1,2}$ Ziwei Xu, ${ }^{1}$ Boris I. Yakobson, ${ }^{1,2}$ Robert J. Young, ${ }^{1,3}$ Ian A. Kinloch, ${ }^{3}$ Shuang Cui, ${ }^{3}$ Libo Deng, ${ }^{3}$ \\ Pascal Puech, ${ }^{4}$ and Marc Monthioux ${ }^{4}$ \\ ${ }^{1}$ Institute of Textiles and Clothing, Hong Kong Polytechnic University, Hung Hom, Hong Kong \\ ${ }^{2}$ Department of Mechanical Engineering and Materials Science and Department of Chemistry, Rice University, \\ Houston, Texas 77005, USA \\ ${ }^{3}$ School of Materials and the Photon Science Institute, University of Manchester, Manchester M1 7HS, United Kingdom \\ ${ }^{4}$ CEMES, UPR 8011 CNRS, Toulouse, France \\ (Received 9 June 2010; published 15 July 2010)
}

\begin{abstract}
Atomistic simulations and a dislocation-based analysis reveal the mechanism of carbon peapod fusion into double-walled nanotubes. They explain the trend of diameter increase for the emerging inner wall, driven by the reduction in its strain energy and the interwall van der Waals energy. Surprisingly, this is also accompanied by the systematic bias in the nanotube chirality, changing from zigzag toward armchair. This prediction agrees well with our experimental data and is further supported by the analysis of earlier observations.
\end{abstract}

DOI: 10.1103/PhysRevB.82.041403

PACS number(s): 61.46.- $-\mathrm{w}, 02.70 .-\mathrm{c}, 61.72 . \mathrm{Lk}$

Double-walled carbon nanotubes (DWNTs) (Ref. 1) are the simplest form of multiwalled carbon nanotubes and as such allow comparisons to be made with the behavior of single-walled nanotubes (SWNTs) (Ref. 2) and enable the study of interactions between concentric graphene tubules. For example, it has been demonstrated recently that there is relatively poor stress transfer between the different walls of DWNTs when deformed in a composite. ${ }^{3}$ Two main techniques have been used in the preparation of DWNTs; ${ }^{1}$ the direct growth using chemical vapor deposition ${ }^{4}$ and the socalled "peapod" route using SWNTs filled with fullerenes ${ }^{5-9}$ where the encapsulated fullerenes coalescence into an inner tube at high temperature. The peapod route is the preferred method for preparing relatively pure DWNTs with welldefined structures. ${ }^{1}$

There has been considerable effort to understand the mechanism of fullerene coalescence and the transformations of $s p^{2}$ carbon networks. ${ }^{8,10-14}$ It is well established that that rotation of a C-C bond in a $s p^{2}$ carbon network, known as the Stone-Wales (SW) transformation, is the key step of such a transformation. ${ }^{10,12,13}$ The calculated barrier of such a bond rotation is as high as 5-9 eV (Refs. 11 and 15) which explains the requirement of high temperature for the formation of peapod-derived DWNTs. ${ }^{9}$ Because of the high barrier, it is impossible to simulate a defect-free DWNT structure by conventional molecular-dynamics simulation due to the limited simulation time (time scale from picosecond to nanosecond). ${ }^{8,14}$ Although a full route from two fullerenes to a short SWNT has been demonstrated in previous studies, the final SWNT formation was predetermined and thus information about the inner-tube chiral angle cannot be determined correctly by these methods. ${ }^{10-13}$

In this Rapid Communication, we study the formation of peapod-derived DWNTs. An atomic simulation successfully reproduces the transformation from peapods into a defectfree DWNT through the SW mechanism. It is found that most of the simulated inner tubes have large chiral angles (e.g., $\theta>20^{\circ}$ ) and detailed theoretical analysis has shown that the preference for large chiral angles is dominated by the driving force of the SW transformation during tube fattening. Through careful analyzing experimental data, we have con- firmed that the abundance of large chiral-angle tubes is in agreement with most experimental observations.

It is well established that the kinetic Monte Carlo (KMC) method can simulate a long-time process by neglecting the thermal vibrations of atoms and considering the process of overcoming barriers directly. In mimicking the $\mathrm{KMC}$ method, we propose a similar method to study the coalescence of fullerenes but by considering instead the energy change in the barrier between two states. In detail, the $s p^{2}$ carbon network is described by the most used second generation Tersoff-Brenner potential in which the van der Waals interactions have been properly incorporated ${ }^{15}$ and the energy of the relaxed initial structure is denoted as $E_{i}$. A C-C bond is then selected randomly and rotated by $90^{\circ}$. The newly obtained structure is relaxed and its local minimum energy is $E_{f}$. The acceptance probability of the structural transformation is 1 if the energy change $\Delta E=E_{f}-E_{i}<0$ or $\exp [-\Delta E / k T]$ if $\Delta E>0$, where $k$ is the Boltzmann constant and $T$ is the temperature. The result from such a simulation would certainly be different from that obtained by a standard $\mathrm{KMC}$ simulation if there were no correlation between the energy barrier, $E_{\mathrm{b}}$, and energy change, $\Delta E$. Fortunately, the well-known Bell-Evans-Polanyi principle in chemistry, ${ }^{16,17}$ that the barrier change is approximately proportional to the formation energy or the energy difference between final state and the initial state, ensures the correlation between $E_{\mathrm{b}}$ and $\Delta E$.

Figure 1 shows formation of a DWNT from the coalescence process of two encapsulated fullerenes (see movie in Ref. 18). The initial configuration is a $(18,0)$ SWNT with two linked $\mathrm{C}_{60}$ molecules inside. A simulation was then performed at $2000 \mathrm{~K}$. During the simulation, a peanutlike structure was formed after several tens of SW steps (b) and the neck between the two $\mathrm{C}_{60}$ 's grows larger $(\mathrm{b} \rightarrow \mathrm{c})$ until a defect-free SWNT is eventually formed (d). This coalescence process is in very good agreement with previous highresolution transmission electronic microscopy observations, ${ }^{14,19}$ which validates the simulation method used in this study. As shown in Fig. 1(e), the small SWNT inside is a chiral $(6,4)$ SWNT. The interwall distance between a $(6,4)$ SWNT and a $(18,0)$ SWNT is $0.37 \mathrm{~nm}$, which 

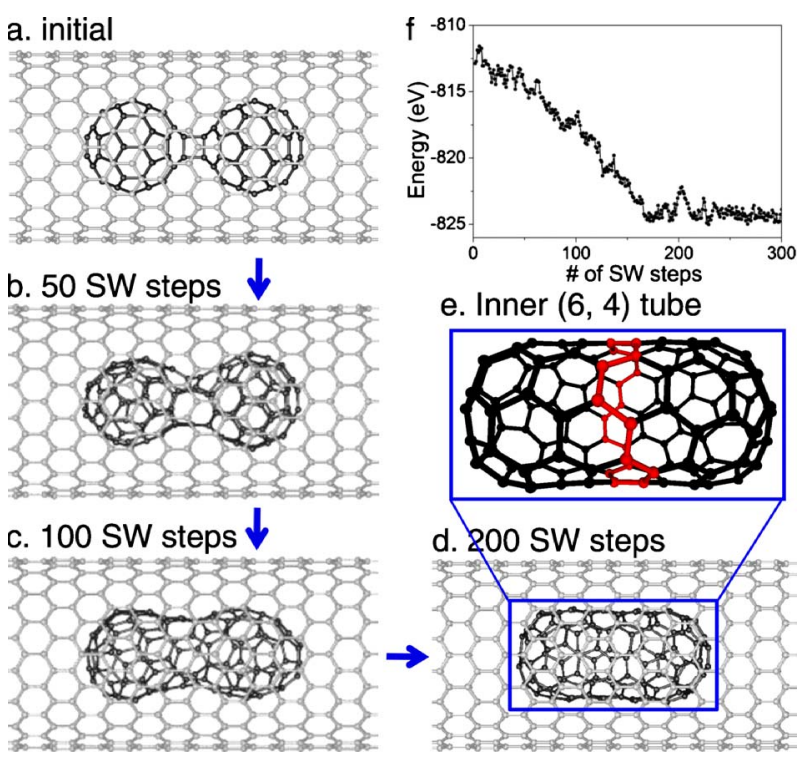

FIG. 1. (Color online) Coalescence of encapsulated fullerenes in a $(18,0)$ SWNT. The two covalent-bond linkages between the two $\mathrm{C}_{60}$ 's (a) were created manually and then a simulation at $2000 \mathrm{~K}$ driven the full coalescence. Steps b, c, and d are snapshots from the simulation and e shows the simulated $(6,4)$ inner-wall tube. A circular chain is marked in e to show the tube chirality. A $13 \mathrm{eV}$ energy drop after coalescence is clearly seen in $\mathrm{f}$.

is in the range of $0.34-0.38 \mathrm{~nm}$ observed for DWNTs ${ }^{20} \mathrm{~A}$ fattening process can be clearly seen during the coalescence from $\mathrm{b} \rightarrow \mathrm{c} \rightarrow \mathrm{d}$. The tube diameter gradually increases and the corresponding length decreases. The main driving force of the coalescence is the reduced curvature together with the energy drop due to defect healing (e.g., there are 24 pentagons in two $\mathrm{C}_{60}$ 's and only 12 survive in a short tube). A continuous energy drop during coalescence can be clearly seen in Fig. 1(f).

The chiral-angle correlation between inner tube and outer tube is a topic of interest. ${ }^{20,21}$ In order to study the chiralangle correlation, we chose four different tubes, $(14,5)$, $(10,10),(18,0)$, and $(19,0)$, whose chiral angles are $14.2^{\circ}$, $30^{\circ}, 0^{\circ}$, and $0^{\circ}$, respectively, as outer tubes. The $(14,5)$ and $(10,10)$ tubes have very similar diameters but the $(18,0)$ and $(19,0)$ tubes' diameters are slightly greater than that of $(10,10)$ by $0.07 \mathrm{~nm}$ and $0.15 \mathrm{~nm}$, respectively. For each outer tube, starting with different configurations, ${ }^{18}$ more than 40 trajectories were performed and the chiral-angle distribution is shown in Fig. 2. For all four outer tubes, the inner-wall tube chiral angles shows very similar distributions, which means there is no chiral-angle correlation between tube inner and outer layers. This is as expected since the van der Waals interaction between tubes depends only weakly on the tube chiral angles. The same conclusion has also been drawn from careful experimental studies..$^{20,21}$

Although there is no chiral-angle correlation between the inner and outer tubes, a significant trend that more than $2 / 3$ of the tubes are found to have large chiral angles of greater than $20^{\circ}$ (Fig. 2 and Ref. 18). To explore the potential mechanism behind this finding, we consider a process of tube fattening as shown in Fig. 3. A bond rotation on the

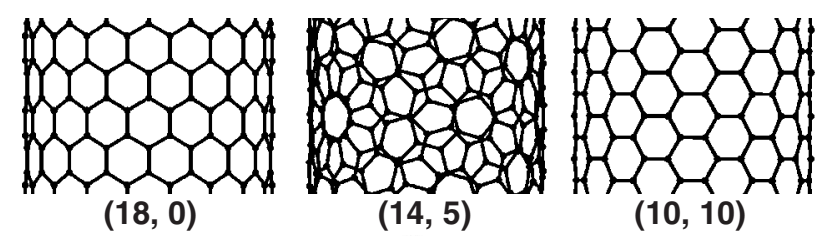

$(18,0)$

$(14,5)$

$(10,10)$

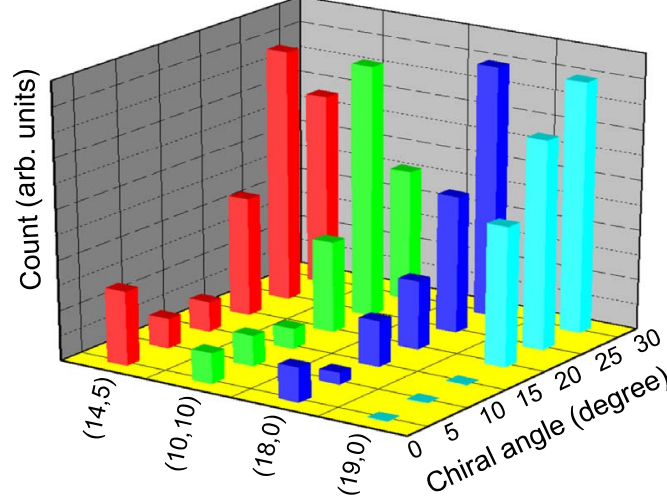

FIG. 2. (Color online) Chiral-angle distribution of the inner tubes of peapod-derived DWNTs. Four different tubes, $(18,0)$, $(14,5),(10,10)$, and $(19,0)$ (not illustrated) were chosen as the outer wall (see also Ref. 18).

SWNT surface turns four hexagons into a $5|7| 7 \mid 5$ formation or a SW defect which can be viewed as two $5 \mid 7$ pairs. A $5 \mid 7$ can be viewed as an edge dislocation in a two-dimensional hexagonal lattice ${ }^{22,23}$ and as an edge dislocation, the motion of $5 \mid 7$ on the tube surface transforms an original tube into another, one Burgers vector, $\mathbf{b}$, away. For example, the separation of the two 5/7's turns a $(8,7)$ nanotube into a $(8,8)$ one, as illustrated in Figs. 3(a)-3(e). Without an external force applied on the tube wall, the only driving force acting on a $5 \mid 7$ is the tube curvature energy change during each SW step. On a tube surface, the distance a $5 \mid 7$ moves in every

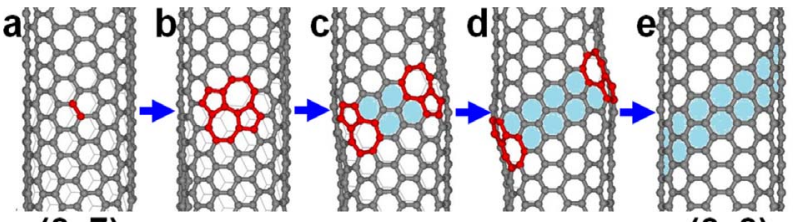

$(8,7)$

$(8,8)$

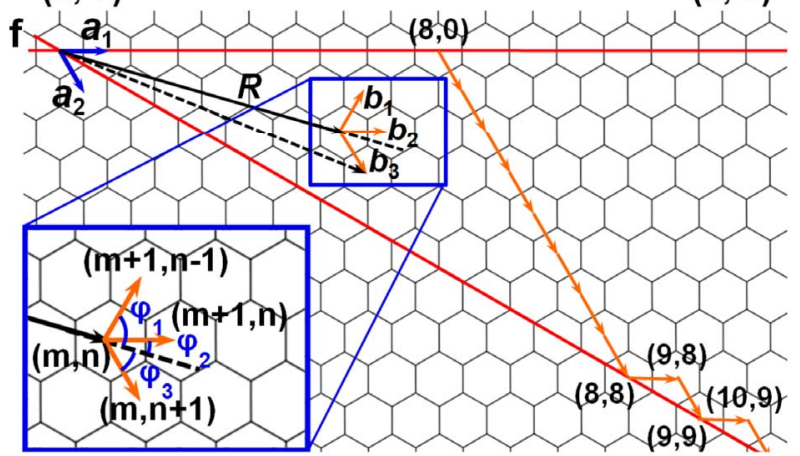

FIG. 3. (Color online) Mechanism of tube fattening. Step a $\rightarrow$ b, a C-C bond rotation creates an SW defects in the nanotube. Steps $\mathrm{b} \rightarrow \mathrm{c} \rightarrow \mathrm{d} \rightarrow \mathrm{e}$, continuous separation of the two $5 \mid 7$ turns on the original $(8,7)$ tube into a fatter $(8,8)$ armchair tube. Diagram $f$, three possible routes for tube fattening. 
step is $b=0.246 \mathrm{~nm} \cdot{ }^{22,23}$ The diameter difference between the two tubes linked by a $5 \mid 7$ is $\Delta D=b *(\cos \varphi) / \pi$, where $\varphi$ is the angle between the Burgers vector and the tube circumference direction. ${ }^{23,24}$ Since the curvature energy of a tube is $\varepsilon=\alpha / D$ per unit length, where $\alpha=10.27 \mathrm{eV} / \mathrm{nm},{ }^{24}$ the curvature energy change is $\Delta \varepsilon \approx \Delta D * d \varepsilon / d D=-\Delta D * \alpha / D^{2}$ per length. The length of a $5 \mid 7$ glide along tube axis direction is $l \sim b * \sin \varphi$ every step and so the driving force acting on a $5 \mid 7 \quad$ is $f=-l \Delta \varepsilon \approx(\alpha / 2 \pi) * \sin (2 \varphi) *(b / D)^{2}$ $=1.63 * \sin (2 \varphi) *(b / D)^{2} \mathrm{eV}$ per SW step. The derived driving force reaches its maximum at $\varphi=45^{\circ}$, which means it is most preferred energetically for a $5 \mid 7$ to glide in a $45^{\circ}$ spiral. For a typical inner tube whose diameter is $\sim 0.7 \mathrm{~nm}$, the maximum driving force on a $5 \mid 7$ is $f=0.20 \mathrm{eV}$, which is greater than the thermal activation energy, $k T$, and thus must play a key role during the tube fattening. On a map of a SWNT [Fig. 3(f)], we can see there are three possibilities of changing tube chirality through a $5 \mid 7$ gliding that have a positive driving forces because of diameter expansion, which are $(m, n)$ to $(m+1, n-1),(m+1, n)$, and $(m, n+1) .^{22}$ Defining the angle between Burgers vector and the tube circumference as $\varphi_{1}, \varphi_{2}$, and $\varphi_{3}$ in Fig. 3(f), it can be easily seen that $\varphi_{1}$ closes to $90^{\circ}$ and $\varphi_{2}$ closes to $0^{\circ}$ and $\varphi_{3}$ is the one nearest to $45^{\circ}$. From above, we can conclude that changing the tube chirality from $(m, n)$ to $(m, n+1)$ is most preferred because of the largest driving force. Such an optimum fattening process, $(m, n) \rightarrow(m, n+1)$, would continuously drive a tube to the armchair type. Once the tube reaches the armchair edge, it has to be changed to another, one Burgers vector away from the armchair edge, i.e., $(m, m) \rightarrow(m$ $+1, m)$ and eventually to the next armchair tube $(m+1, m$ $+1) \ldots$ as shown in Fig. 3(f). This analysis shows that tube fattening is critical for the large chiral-angle inner-tube formation. Such a trend can also been seen in the simulations in Fig. 2. For the largest outer tube, $(19,0)$, we studied, no inner tube whose chiral angle is smaller than $15^{\circ}$ was seen.

The positions of the Raman bands in the low-frequency radial breathing mode (RBM) region of the spectrum enable identification of the chiralities of the tubes in resonance. ${ }^{2}$ Conversion from peapods to DWNTs can be monitored as the formation of the inner walls leads to the appearance of a population of extra RBMs in the $220-400 \mathrm{~cm}^{-1}$ region ${ }^{1,3,5,9}$ (see Ref. 18). Pfeiffer and co-workers ${ }^{1,6,7}$ undertook a comprehensive study of the RBMs from inner walls of peapodderived DWNTs. They showed that families of peaks are usually obtained for inner tubes with a particular value of $(n, m)$ since the same inner tube can be accommodated in different diameter outer ones. Abundance of large chiralangle inner tubes such as $(6,4),(6,5)$, and $(7,5)$ has been clearly seen but the laser energy used was not high enough to observe armchair inner-wall tubes such as $(5,5)$ or $(6,6)$. $^{1,25}$

Figure 4(a) shows Raman spectra obtained in the innerwall $220-400 \mathrm{~cm}^{-1}$ region of the DWNTs using three different laser excitation energies. The assignments of the main RBMs are indicated and for comparison, spectra in the same region are also presented for high-pressure carbon monoxide (HiPco) processed SWNTs. The most prominent RBM in the DWNTs with the $1.49 \mathrm{eV}$ laser can be assigned ${ }^{26}$ to the $E_{11}^{S}$ transition of the small-diameter high chiral-angle $(5,4)$ tube at $384 \mathrm{~cm}^{-1}$. Nanotubes as small as this are not found in
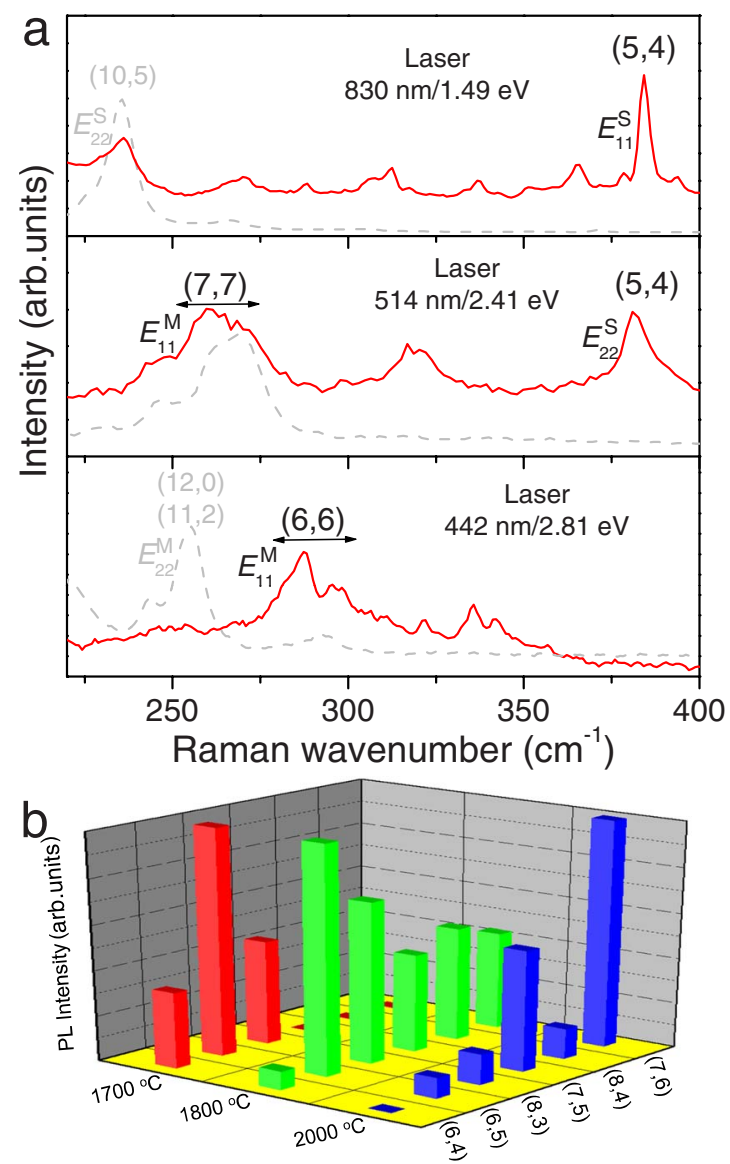

FIG. 4. (Color online) (a) Raman spectra in the inner-wall RBM region obtained using different indicated laser excitation energies. The solid line is for the peapod-derived DWNTs and the dashed line is for HiPco SWNTs. The assignment of the major peaks is indicated. (b) Relative PL intensity of the inner wall of DWNTs made from peapods by thermal annealing at different temperatures $1700{ }^{\circ} \mathrm{C}, 1800{ }^{\circ} \mathrm{C}$, and $2000{ }^{\circ} \mathrm{C}$, respectively. The PL intensities at each temperature have been rescaled for clarity. (PL data were extracted from Ref. 9).

HiPco and the only RBM seen for the HiPco is from $(10,5)$; this RBM is relatively weak for the DWNTs. The spectrum for the DWNTs obtained using the $2.41 \mathrm{eV}$ laser shows two main sets of RBMs. One is at around $380 \mathrm{~cm}^{-1}$ corresponding to the $E_{22}^{S}$ transition of the $(5,4)$ tube. Another group in the region $250-280 \mathrm{~cm}^{-1}$ is mainly contributed by the $(7,7)$ tube, ${ }^{26}$ which belongs to family 21 tubes, ${ }^{2}$ inside outer tubes of different diameter. The spectrum obtained using the highenergy $2.81 \mathrm{eV}$ laser is particularly significant in that it shows a family of peaks in the $280-300 \mathrm{~cm}^{-1}$ region mainly from the $(6,6)$ tube. ${ }^{26}$ HiPco does not have an RBM in this region and shows only a peak corresponding to the low chiral-angle $(12,0) /(11,2)$ RBMs. This study of the Raman RBMs from the inner walls using the different laser excitation energies has demonstrated clearly that small-diameter high chiral-angle inner-wall tubes are abundant in our peapod-derived DWNT material.

Photoluminescence (PL) gives an indication of the relative abundance of inner walls of different chiralities. Muramatsu et $a l .{ }^{9}$ have recently reported bright PL from the inner 
walls of peapod-derived DWNTs treated at high temperatures. Their data are reproduced in Fig. 4(b) where the intensity of the PL for each tube is shown for a group of tubes ranging from $(6,4)$ to $(7,6)$ (i.e., increasing in diameter) for the different annealing temperatures. A number of important conclusions can be made that confirm the predictions of our theory. First, there is a clear tendency for the tubes to fatten since the majority of the population moves toward $(7,6)$ as the temperature is increased. Second the most populous tubes are those with the highest chiral angles [i.e., $(6,5)$ and $(7,6)]$. There is no signal of inner armchair tubes because of the major drawback of PL-it is only capable of "seeing" semiconducting nanotubes.

In conclusion we have demonstrated clearly from our simulation that the development of the inner walls in peapodderived DWNTs takes place though the cooperative motion of Stone-Wales defects leading to a preference for the innerwall nanotubes to have very high chiral angles. This is confirmed from both our own experiments upon the Raman RBMs from the inner walls using both high- and low-energy lasers and data in the literature. It implies that there is an abundance of metallic armchair nanotubes in the inner walls of DWNTs leading to potential new applications.

Work at PolyU was supported by PolyU internal foundation (Grants No. 1-ZV3B, No. A-PD1U, and No. A-PH93); work at Rice was supported by the National Science Foundation under Grant No. CBET-0731246.
${ }^{1}$ R. Pfeiffer, T. Pichler, Y. Ahm Kim, and H. Kuzmany, in Carbon Nanotubes, edited by A. Jorio, G. Dresselhaus, and M. S. Dresselhaus, Topics in Applied Physics Vol. 111 (Springer, Berlin, 2008), p. 495.

${ }^{2}$ M. S. Dresselhaus et al., Phys. Rep. 409, 47 (2005).

${ }^{3}$ S. Cui et al., Adv. Mater. 21, 3591 (2009).

${ }^{4}$ T. Yamada et al., Nat. Nanotechnol. 1, 131 (2006).

${ }^{5}$ B. W. Smith et al., Nature (London) 396, 323 (1998).

${ }^{6}$ B. W. Smith et al., Chem. Phys. Lett. 321, 169 (2000).

${ }^{7}$ R. Pfeiffer et al., Nano Lett. 7, 2428 (2007).

${ }^{8}$ X. Li et al., Nano Lett. 7, 3709 (2007).

${ }^{9}$ H. Muramatsu et al., Small 5, 2678 (2009).

${ }^{10}$ Y. Zhao, B. I. Yakobson, and R. E. Smalley, Phys. Rev. Lett. 88, 185501 (2002).

${ }^{11}$ Y. Zhao, Y. Lin, and B. I. Yakobson, Phys. Rev. B 68, 233403 (2003).

${ }^{12}$ S. Han, M. Yoon, S. Berber, N. Park, E. Osawa, J. Ihm, and D. Tománek, Phys. Rev. B 70, 113402 (2004).

${ }^{13}$ I.-H. Lee et al., Appl. Phys. Lett. 88, 011913 (2006).

${ }^{14}$ E. Hernández et al., Nano Lett. 3, 1037 (2003).

${ }^{15}$ D. W. Brenner et al., J. Phys.: Condens. Matter 14, 783 (2002).
${ }^{16}$ R. P. Bell, Proc. R. Soc. London, Ser. A 154, 414 (1936).

${ }^{17}$ M. G. Evans et al., Trans. Faraday Soc. 31, 875 (1935).

${ }^{18}$ See supplementary material at http://link.aps.org/supplemental/ 10.1103/PhysRevB.82.041403 for a movie of two $\mathrm{C}_{60}$ 's coalescence, summary of simulated inner tubes, initial configurationdependent $\mathrm{C}_{60}$ 's coalescence and RBM spectra of the original SWNTS and peapod-derived DWNTs.

${ }^{19}$ S. Bandow et al., Chem. Phys. Lett. 337, 48 (2001).

${ }^{20}$ K. Hirahara, M. Kociak, S. Bandow, T. Nakahira, K. Itoh, Y. Saito, and S. Iijima, Phys. Rev. B 73, 195420 (2006).

${ }^{21}$ A. Hashimoto, K. Suenaga, K. Urita, T. Shimada, T. Sugai, S. Bandow, H. Shinohara, and S. Iijima, Phys. Rev. Lett. 94, 045504 (2005).

${ }^{22}$ B. I. Yakobson, Appl. Phys. Lett. 72, 918 (1998).

${ }^{23}$ F. Ding et al., Nano Lett. 7, 681 (2007).

${ }^{24}$ O. Gulseren, T. Yildirim, and S. Ciraci, Phys. Rev. B 65, 153405 (2002).

${ }^{25}$ R. Pfeiffer et al., Phys. Status Solidi B 245, 1943 (2008).

${ }^{26}$ The $(n, m)$ assignments were undertaken using values for $E_{11}^{M}$ and $E_{i i}^{S}$ determined from tight-binding calculations (e.g., confirmed with Ref. 2). 